\title{
Human-Centric Functional Modeling and the Convergence of Science
}

\author{
Andy E. Williams ${ }^{1 *}$ \\ ${ }^{1}$ Nobeah Foundation, Nairobi, Kenya \\ *awilliams@nobeahfoundation.org
}

\begin{abstract}
This paper explores Human-Centric Functional Modeling as a universal modeling methodology with the potential to be used for representing all systems both conceptually and functionally, and it explores what capacity for convergence in our understanding of systems means in terms of Human-Centric Functional Modeling, how that capacity might be quantified, and why Human-Centric Functional Modeling as a methodology might maximize our collective capacity to achieve convergence in our understanding across all sciences and across the systems that those sciences study.
\end{abstract}

\section{Introduction}

Network scientists have long understood that the behavior of systems can be represented in terms of networks [1], and it is this idea that has recently used to provide mathematical rigor to a startlingly ancient unifying theme that potentially underlies our innate understanding of all systems. The surprise is that it is looking back to the ancient philosophy which embraces this unifying theme that might hold the greatest promise to reveal deep new insights in the future about our universe and all of this existence, as well as holding the promise to lay the foundations for a disruptive leap forward in a great many of the disciplines of modern science that we've created to study the world around us. This philosophy is captured by the emerging science of Human-Centric Functional Modeling or HCFM, which leverages this concept of networks to represent all systems through analogy with the cognitive system.

Human-Centric Functional Modeling represents the behavior of systems in terms of motion through "functional state spaces" similar to the one the human cognitive system can be represented as navigating. Every system can potentially be understood as transitioning from one functional state or some combination of functional states to another, where the functional states within the system's behavior belong to either a closed set or to an open set to which new states might be added or from which existing states might be removed. Therefore every system can potentially be understood as having behavior confined to the set of functions through which it might transition from any one functional state or combination of states to the next. Without even knowing the physical or virtual structures which might correspond to these functional states, and without even knowing the mechanisms through which the functions transitioning between these states might be implemented, it is then possible to represent the behavior of the system in terms of whatever properties characterize its motion through this network of functional states.

From the perspective of Human-Centric Functional Modeling human cognition moves through a functional state space in which the functional states are concepts, or in other words human cognition moves from one concept to another, using reasoning to do so. Where an ontology defines a closed set of concepts and the set of reasoning processes that connect each concept, HCFM defines an open network to facilitate the definition of every possible concept and every possible reasoning process connecting each concept. This graph then represents the complete human meaning of each concept and each reasoning process. If the graph of the functional state space of the cognitive system (the conceptual space) represents all the human meaning of each concept and each reasoning process, it is therefore a complete semantic model. 


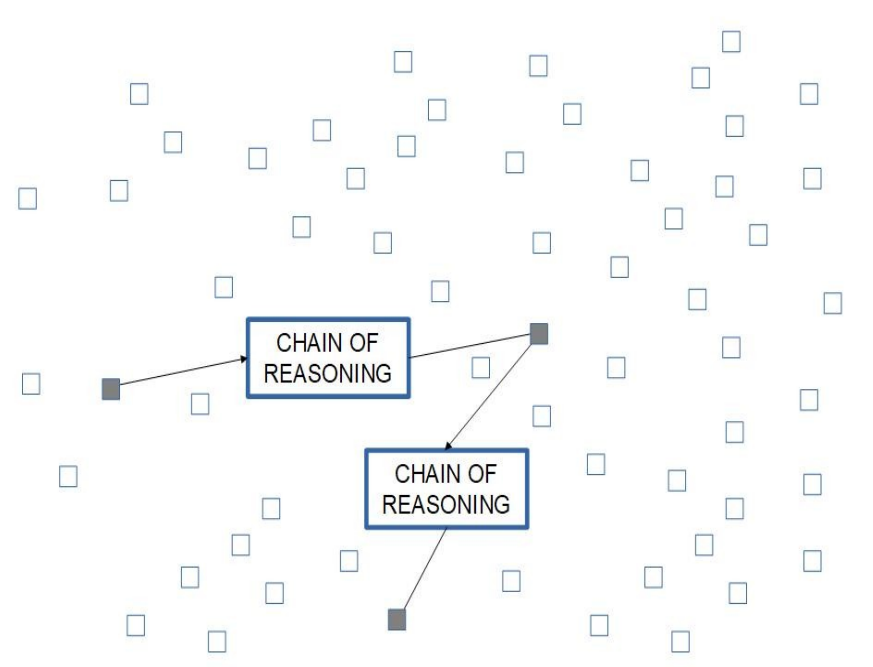

Figure 1: conceptual space as a graph of concepts (the functional states of the cognitive system) connected by reasoning processes.

If the conceptual space is a complete semantic model, then by analogy the graph describing the functional state space of any other system represents the complete functional meaning of each externally observable functional state and of each externally observable process connecting the functional states of that system and is therefore a complete semantic model of the observable behavior of that system as well.

\section{Constructs in Conceptual Space - Zoom In}

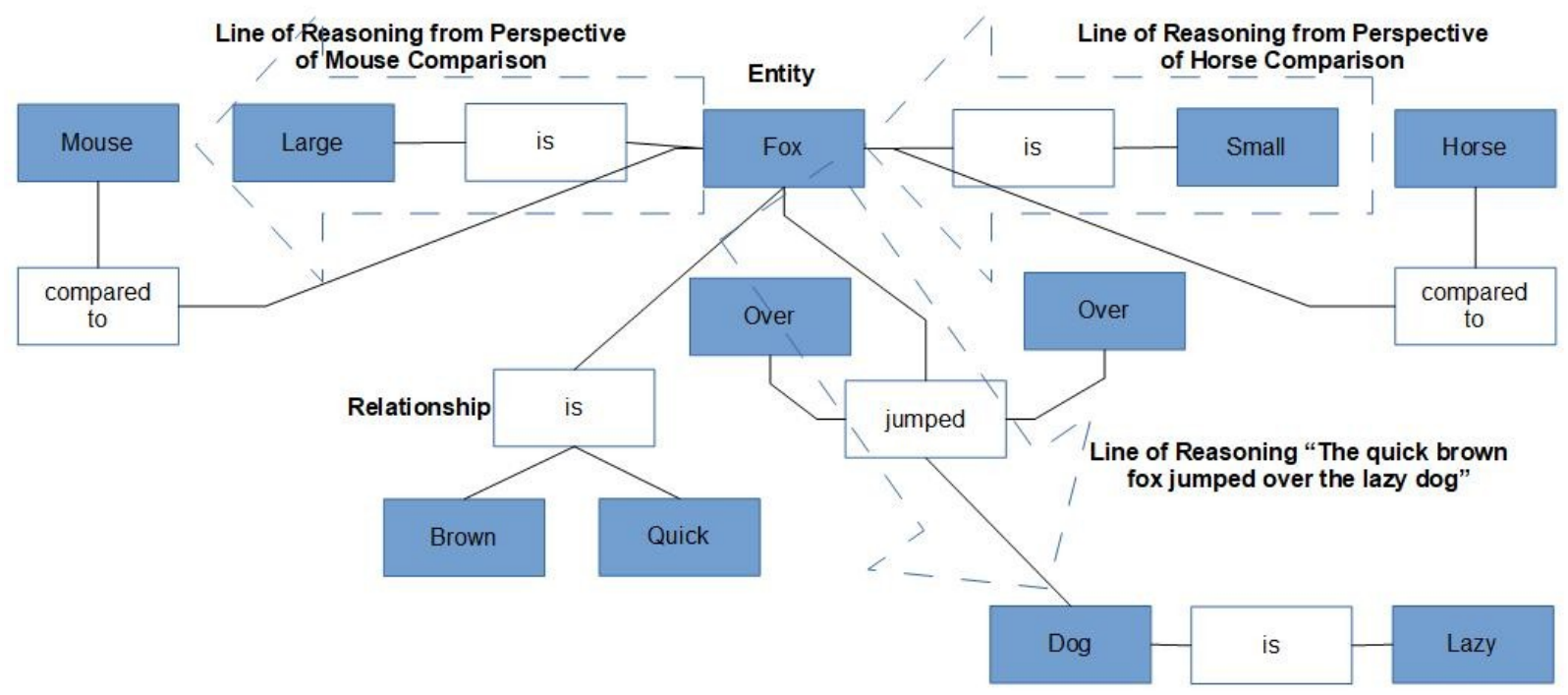

Figure 2: Representation of actual concepts and reasoning processes in conceptual space.

If the graph describing the functional state space of the system is a complete semantic representation of the observable behavior of the system, then each functional property of the system must have an expression of meaning that can be represented in the graph. Since the graph is a mathematical construct we assume that representations in that graph are mathematical representations. This implies that each system property has a mathematical expression in the graph. 
As an example, "complexity" as a property of a problem in conceptual space is hypothesized to be represented by the distance in conceptual space that the cognitive system must navigate to solve the problem, multiplied by the linear density of concepts that the cognitive system must move through in doing so. Similarly, considering homeostasis as another system, describing the homeostasis process of organisms as also moving through its own functional state space, then the complexity of any problem of achieving homeostasis is hypothesized to again be represented by the distance in the homeostasis functional state space that the homeostasis system must navigate to solve the problem, multiplied by the linear density of functional states that the homeostasis system must move through.

For any system, the characteristics of this movement within its resulting network of functional states connected by its functions (processes) are then expected to define properties of the system such as complexity of problems or of solutions, or the problem-solving ability of the system. Wherever these system properties are involved in deducing theoretical relationships between these functional states and processes, these properties are hypothesized to set constraints for system behavior. As an example, a petri dish full of single-celled organisms is neither observed nor expected to have the problem-solving ability to organize themselves into a bird in order to solve the problem of flight. In Human-Centric Functional Modeling their level of problem-solving ability is a property of the group as a system.

These constraints might then determine which problems the system is capable of solving and what solutions the system is capable of solving them with, along with determining which theories about fundamental aspects of the system can be valid. As another example, representing matter in any region of the physical universe as a functional state space containing a network of functional states connected by transition processes represented by some composition of the four fundamental forces, there might be some pairs of states between which there is no path within the problem-solving ability of the universe. Achieving these transitions are problems which this model suggests cannot be solved within this observable universe.

In summary, Human-Centric Functional Modeling defines a universal approach for modeling the behavior of all systems in terms of motion through "functional state spaces" similar to the functional state space the human cognitive system can be represented as navigating. Since the external (conscious) functions of cognition can by definition be conceptualized, since all conscious cognitive states and functions are represented in the graph of functional state space, and since all properties of the cognitive system in executing those conscious functions are defined by that graph of functional state space, then a mathematical expression can potentially be deduced to describe any property of cognition in terms of that graph. Because the structure of one open functional state space is potentially the same as the structure of any other open functional state space, then using the functional state space of the cognitive system to deduce mathematical expressions representing the properties of cognition as a system potentially enables the same mathematical expressions to be used to define equivalent properties in vastly different systems, so that all equivalent properties such as "complexity" in other systems might be intuitively understandable through comparison with properties of cognition.

\section{Maximizing Capacity to Understand Systems Complexity}

What makes HCFM potentially so important is its potential to maximize our capacity as individuals to understand and navigate the complexity of any system, through facilitating an exponential increase in our collective ability to solve any problems in general in relation to any system, where this model suggests that ability might not be reliably achievable otherwise. Since all systems, not just cognition, might also be represented in terms of a network in a functional state space in this same way as cognition, and assuming all functional properties of any system might be represented through analogy with their representation in the graph of the functional state space of cognition, then all functional 
properties of any system might be represented in terms that are analogous to the way we understand our own minds, an understanding we can potentially achieve without any technical language or external tools by simply looking inwards. Put in different terms, if the properties of the cognitive system are defined by the graph of functional state space, and if all subsets of the graph of conceptual space (any subset of concepts and reasoning) can by definition be conceptualized, then any properties of the cognitive system can be conceptualized. If an open functional state space is a complete representation of the states and behavior possible for the system it represents, and if the representation of any open functional state space as a graph is the same for any system, then any property that can be represented in any open functional state space is a property that can be represented in conceptual space (the open space of concepts representing the functional state space of the cognitive system), and therefore any such property can by definition can be conceptualized. If the description of any state or behavior is invariant between open functional state spaces as hypothesized, the expressions defining properties for the cognitive system within HCFM also apply for other open systems.

In HCFM reasoning and understanding are processes through which the cognitive system navigates between concepts, the difference between the two being that reasoning is represented as being consciously directed, while understanding is represented as being passively received. In abstract terms concepts are just functional states of the cognitive system; any reasoning or understanding are processes through which the cognitive system transitions between functional states, and any property of the cognitive system is represented in terms of the graph of these functional states and processes (behaviors). The maximum subset that can exist for any set is the subset containing all of that set, and any approach that enables any functional state, behavior, or properties of any system to be conceptualized is one that enables them to be understood. Any approach that enables all of the functional states, behaviors, and properties of any system to be understood is one that maximizes human capacity to understand that system from the functional perspective.

Having proposed distance in conceptual space multiplied by the linear density of concepts as an expression for the complexity of any reasoning process, then if complexity is a property of human cognition and if the maximum number of systems (all systems) can be understood through understanding the human cognitive system and through understanding the analogy of those systems with the cognitive system using HCFM, then from the fact that complexity is one of the properties of cognition that can be understood, it follows that HCFM provides a universal approach to modeling systems that can potentially maximize human capacity to understand and navigate the complexity of all other systems as well. From another perspective, according to Gödel's completeness theorem [9], if the existence of this hypothetical functional state space capable of describing any system is true, then it is provable. If the existence of this hypothetical functional state space capable of describing any system is true and provable, and if in addition each functional state space provides a model of the system it describes, where that model maximizes the ability of human cognition to understand that system and its properties including complexity, it follows that HCFM maximizes human capacity to understand and navigate the complexity of any system.

In other words HCFM facilitates a kind of biomimicry in which the human organism is represented in terms of abstract mathematical spaces that can be used to define simple expressions to represent properties like "complexity" for human systems like cognition, where the same mathematical spaces can be used to represent other systems. The underlying equivalence of the representations of certain properties and their applicability to potentially very different systems therefore allows solutions that have used to solve complexity in this natural human organism to be "mimicked" or otherwise reused in addressing complexity in other systems. Since these include "wicked problems" in human groups like 
achieving sustainability [5], and since these other systems include the entire physical universe [3], the potential applications of HCFM are far reaching.

In particular, for any system in the special category of "adaptive problem-solving systems" which includes human cognition, it is hypothesized that:

- The category of systems with general problem-solving ability exists, where general problemsolving ability can be defined as a property of a system in its functional state space.

- Any system that navigates its functional state space with general problem-solving ability belongs to the category of all systems that do.

- Categories are defined such that any property defined in any functional state space belonging to a given category, is a property that applies to all functional state spaces for all systems of the same category.

- Any property defined in conceptual space for the cognitive system, as a system belonging to the category of those having general problem-solving ability, is a property that applies to all functional state spaces of systems having general problem-solving ability.

- A theory is a set of claims. Any set of claims and therefore any theory regarding the category of systems with general problem-solving ability can be expressed in terms of properties belonging to all systems with general problem-solving ability.

In summary, it is hypothesized that if a system can be represented by an open functional state space and has the property of general problem-solving ability in that functional state space, then any theory that can be proved on the open functional state space in terms of the properties characteristic of any system with general problem-solving ability, is a theory that applies to all such systems. The importance of this hypothesis is that it predicts similar phenomena in wildly different systems (for example predicting a "big bang" in both cognition and in physical space at well-defined phase transitions [3]) simply due to this equivalence in functional state space.

\section{A New Kind of Convergence}

The ability to solve problems using any given reasoning process and the ability to take that reasoning from one subject matter domain and to reuse it in another is not necessarily a useful definition of convergence. The question of "how many fairies can fit on the head of a pin" might have solutions that are "correct" in terms of being consistent with whatever folk tradition is considered to be authoritative, but whether or not those solutions are useful in deriving insights in other fields from perspectives outside that tradition is determined by whether those solutions are "true".

In conceptual space, or in any other functional state space, the capacity for convergence is hypothesized to be the capacity to use a truth from one subject area to discover truth in another. In practical terms, where a problem definition or reasoning provides a solution, and where that problem definition or reasoning have been determined to be "true" in a relative or absolute sense, this means the capacity to take that truth from any one subject, perhaps mathematics, and reusing it to discover truth in any other subject, perhaps sociology. Since subject areas correspond to regions in conceptual space, this translates to the capacity to use truth from one region in conceptual space to discover truth in another. A mathematical expression for this convergence is hypothesized here to be that capacity for convergence is equal to the volume of truth that can be discerned per unit volume of functional state space in the region of the target concept in conceptual space, divided by the volume of truth that can be discerned per unit volume of functional state space in the region of the initial concept in conceptual space. The purpose of attempting to quantify convergence in this way is to be able to identify and compare the patterns by which convergence might be increased, so that a significant or even an exponential increase 
in convergence might be achievable. Since the expression for convergence depends on truth, this requires an understanding of what relative and absolute truth means in functional state space.

In functional state space truth has two representations, one is relative truth which has been defined as a signal [7]. Any given reasoning processes in conceptual space, or any given process in any other functional state space, has been represented as a channel that conveys truth as a signal with some degree of error that is hypothesized to be given by information theory [7]. In other words, in conceptual space the relative truth of one claim can be defined in terms of the self-consistency of the group of claims contained in the generalization of that claim.
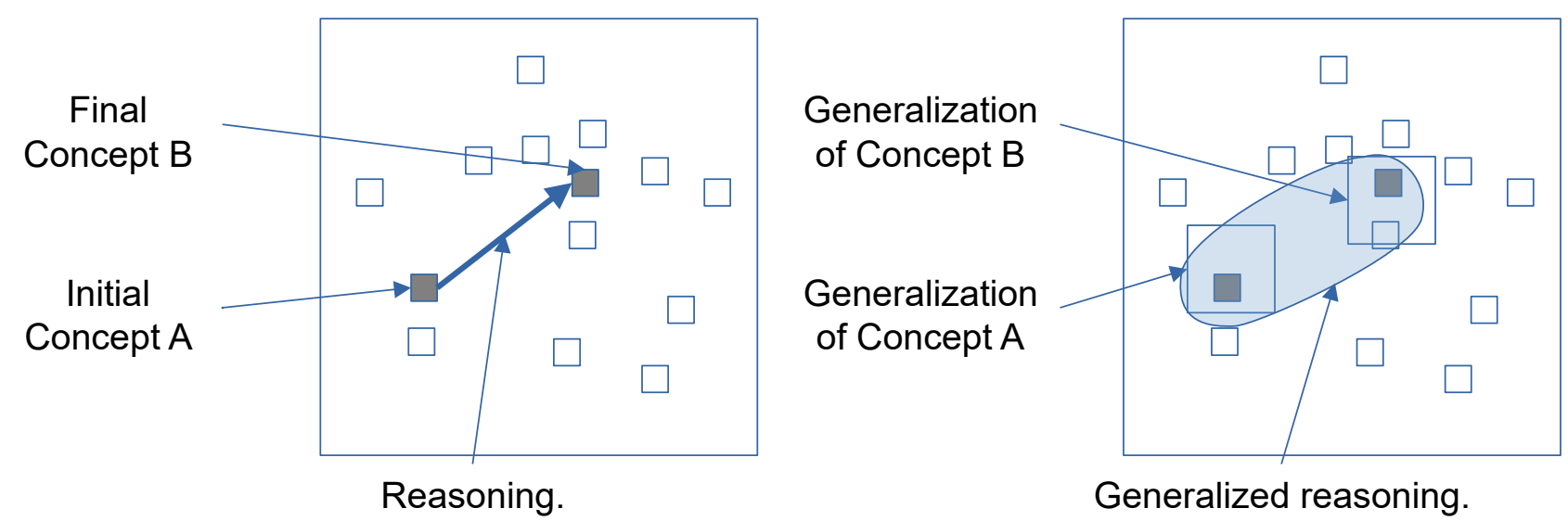

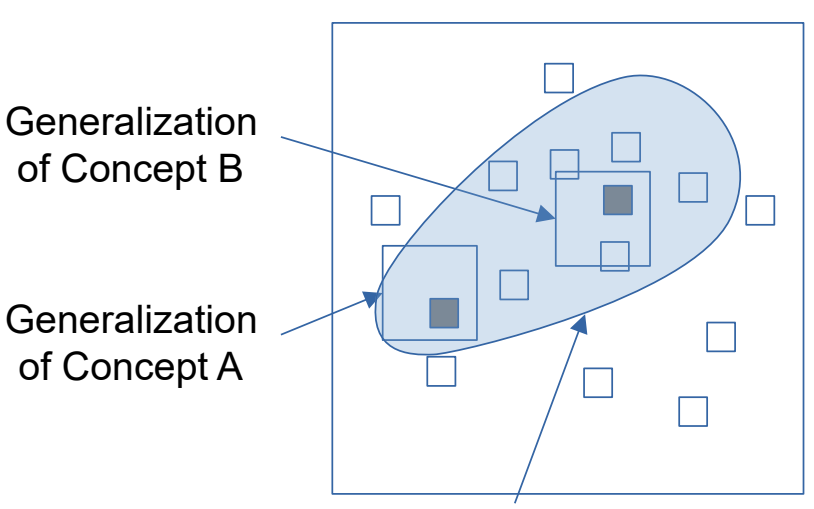

Generalized reasoning.

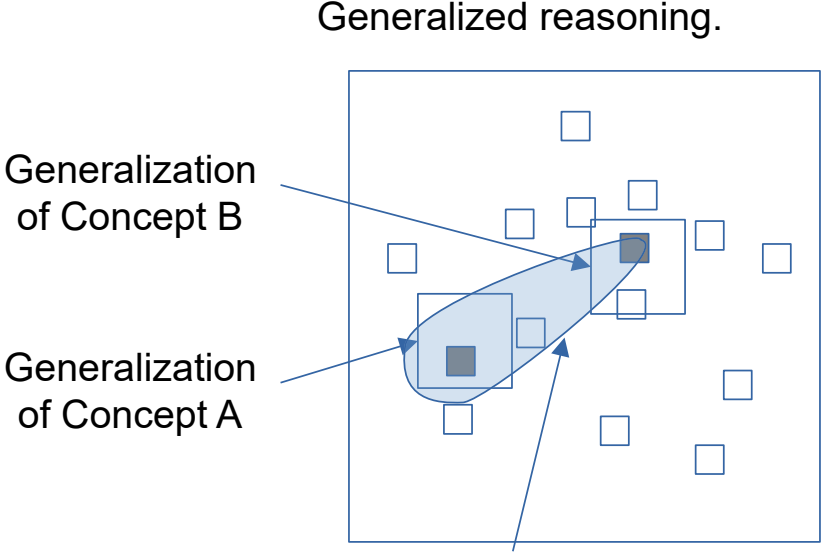

Generalized reasoning.

Figure 1: Truth in Functional State Space. Top left: reasoning representing a claim of unknown truth that connects some concept $A$ and some concept B. Top right: generalized reasoning representing a claim that is true in the sense that all reasoning processes that can be generalized as belonging to the same set are consistent in tracing a path between generalizations of the same concept $A$ and B. Bottom left: generalized reasoning representing a claim that is partially true in the sense that some reasoning processes that can be generalized as belonging to the same set are not consistent and trace a path from the generalization of concept A but do not end up within the generalization of concept B. Bottom right: generalized reasoning representing a claim that is completely true but that is only part of the truth in the sense that all reasoning processes that can be generalized as belonging to the same set are consistent in tracing a path between generalizations of the same concept A and B, but some of the paths between those concepts are outside that generalized reasoning process.

Another representation of truth is absolute truth. If a functional state space can be defined in any Euclidean or in any positively or negatively curved space, any geometry defined in that functional state 
space is hypothesized here to represent an absolute truth in that all statements about that geometry that are true, must be contained within that functional state space, and therefore must be provable within that geometry [10]. These statements regarding the definition of absolute truths in functional state space remain to be proved. However, once relative or absolute truths can be defined in any functional state space then relationships can be defined between those truths. One set of relationships between truths are (geometric) transforms in functional state space from one truth to another. Define a transform to be "true" if all entities that are "true" in the absolute or relative sense are also transformed to be true in the absolute or relative sense respectively.

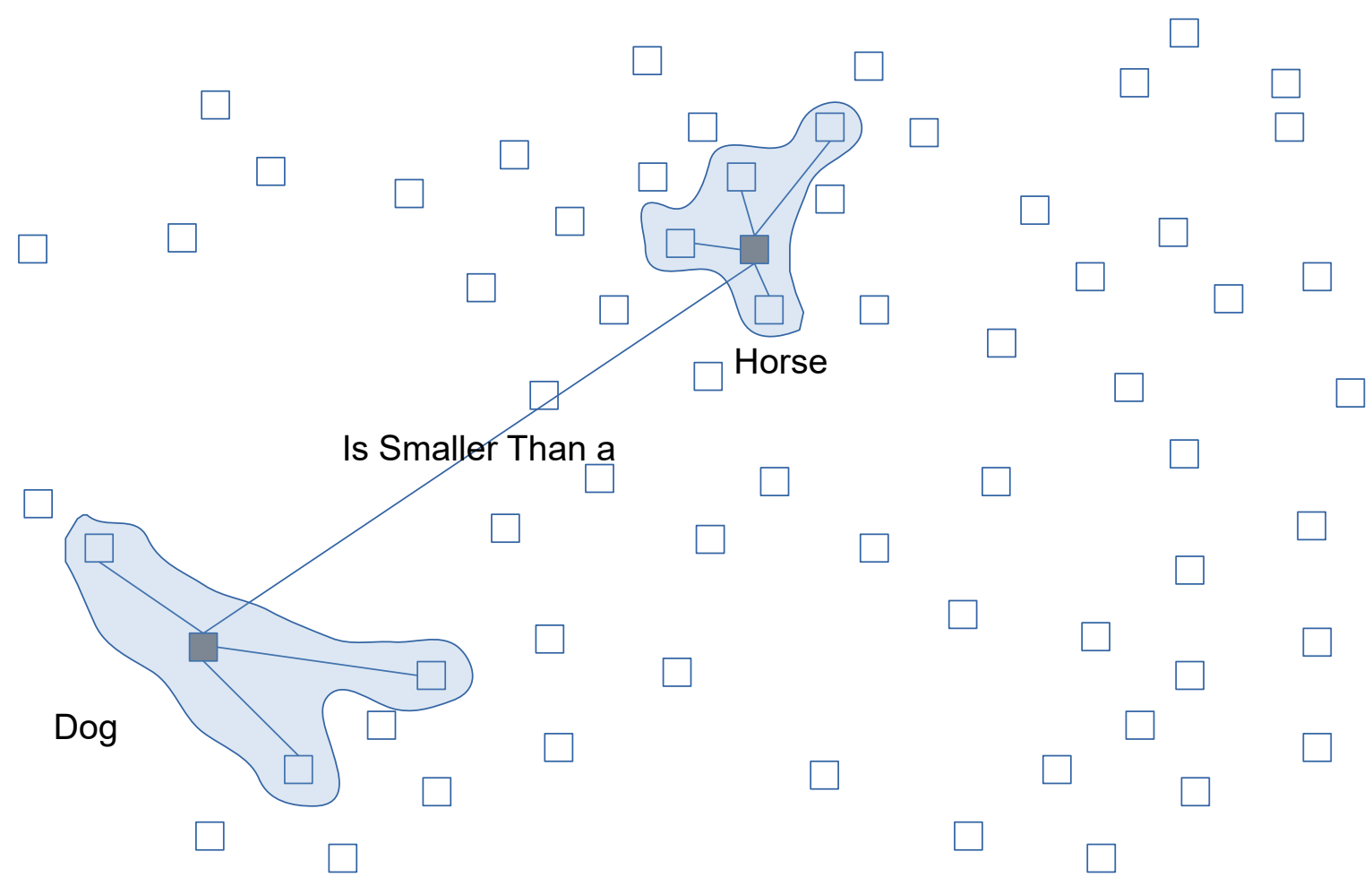

Figure 2: A geometry in conceptual space encompasses a set of claims. This geometry might represent the claim "a dog is smaller than a horse".

Then any reasoning that is "true" in terms of being a solution to any one problem might be generalized through such transforms to any other problem where it applies ("applies" in the sense that it is also true). 


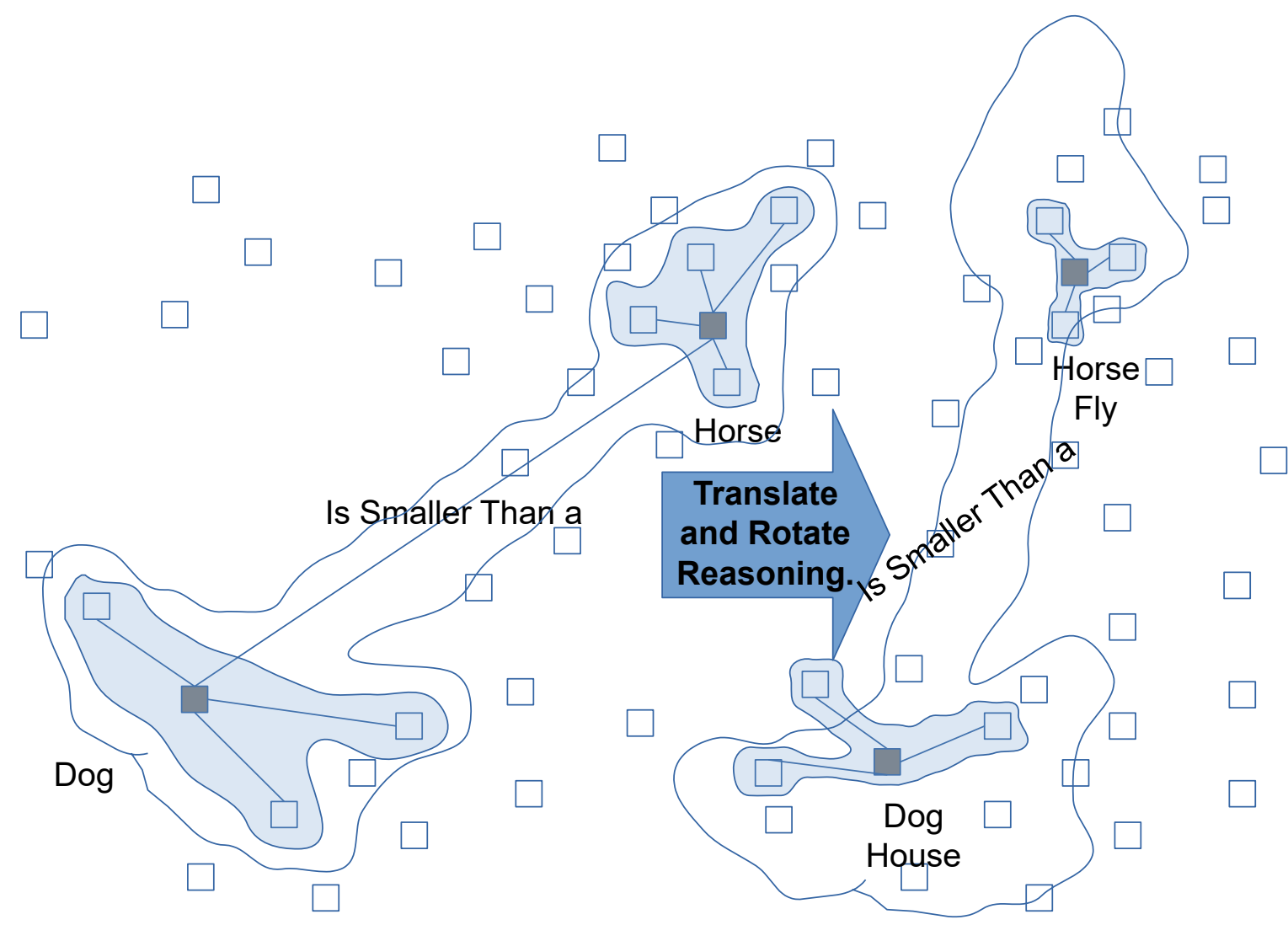

Figure 3: Some geometric transformations might be applied to the geometry of a claim in conceptual space (e.g. the geometry might be translated and rotated) in conceptual space and applied to a new set of concepts in that new region of conceptual space. Some geometries when transformed to a different region of conceptual space are no longer "true" as in the above case in which the geometry of the claim "a dog is smaller than a horse" is transformed to begin at the concept "dog house" and to end at the concept "horse fly". In this second case the claim does not encompass the required initial and target concepts and therefore is not "true".

Geometries might be defined independently of concepts themselves in conceptual space to represent absolute truths. However, if the resolution of functional state spaces is given by the resolution of the functional states in those spaces, and if the equivalence of open functional state spaces suggests that the uncertainty principle which governs physical space also has some equivalent in other functional state spaces, then every known functional state space is limited in the resolution at which it is able to represent the truth of a geometry. Consider a square region in some functional state space (the functional state space representing matter, or the functional state space representing cognition). Depict functional states in these functional state spaces as being separated and as interacting through processes that connect empty space. In the case of the cognition, part of the empty regions of the functional state space in the geometry of that square might be occupied by a higher order cognition with a higher density of concepts in conceptual space. However, in the absence of that higher order cognition these functional states do not occur and interactions between them do not exist. In the case of matter in the physical universe, the empty regions of the functional state space in the geometry of that square might be occupied by a higher order system, such as dark matter might be speculated to be. In both cases, the truth described by a geometry is a limit of truth. But it is a limit that potentially cannot be reached. 
The set of all systems that can potentially be represented by open functional state spaces includes both cognition, consciousness, as biological processes that are adaptive problem-solving systems acting on an unbounded set of states. It also includes inanimate systems that are adaptive problem-solving systems acting on an unbounded set of states, including the physical universe. As mentioned however, though these states are unbounded, they are limited in resolution to the characteristics of the systems themselves. Since software represents the automation of reasoning processes, and since hardware represents the automation of physical processes, then all software systems or hardware that might be navigated by a cognitive system, where the functionality is open, can also potentially be represented by open functional state spaces.

Concepts are a specific category of functional state. Assume this category is related to some capacity of that functional state to store information. A sensory state (that is, a state defining the tactile, auditory, visual, gustatory, or olfactory systems) can be conceptualized, and can therefore fit within a conceptual state. By contrast, from the observation that our sensory perception cannot reliably sense any given concept, we have no assurance that a concept can necessarily fit into a sensory state. However, any concept can come into our conscious self-awareness, and therefore can fit into an awareness state of the consciousness system. The reverse is also not necessarily true (not all awareness's can be conceptualized, so it follows that an awareness does not necessarily fit into a concept). From this simple experiment in first person observation we can deduce that somehow concepts as functional states are more general than sensations, and that each awareness as a functional state is somehow more general than a concept. The takeaway is that different amounts of information or different sizes of "truths" might exist in each functional state space. For example, all of these functional state spaces (the sensory space, the emotional space, the conceptual space, and the awareness space) can contain truths. It is unknown which functional state space, if any, can accommodate all truths. While we can use the analogy with other functional state spaces to define properties like "complexity" for the tactile space or for other functional state spaces that are simpler than the conceptual space, and while our consciousness can be aware of those functional states, in some sense there must be a limit to the degree to which the truth of functional states, behaviors, or properties can be determined in each functional state space, and these limits must have some relationship between functional state spaces if one functional state is able or not able to contain the truths of another. Some such limits might be the resolution in each functional state space at which we can determine truth and the size of truth in each functional state space that we can perceive.

The implications of exponentially greater capacity for convergence in science, and therefore of exponentially greater capacity to reapply truths, are far reaching. One is that if the truth of any biological system can be understood in terms of it functioning as having general problem-solving ability in some functional state space, then all biological or non-biological systems with general problem-solving ability in some functional state space might be understood the same way [2]. If the origin of cognition and other biological processes representing adaptive problem-solving systems can be defined in functional state space, this understanding might also apply to the origin of the physical universe as an adaptive problem-solving system [3]. If the "big bang" is a pattern in physical space, it might also be a pattern in cognitive space as a functional state space, as suggested by one analysis [4]. If any solution that creates self-assembling, self-sustaining processes that massively increase impact on collective outcomes per unit of resources is proved to exist in the functional state space of living things, that solution might be able to be replicated in the functional state space of civilizations to solve equivalent problems required radically increasing outcomes per unit of resources, like poverty, climate change, or the other sustainable development goals [5]. 
Human-Centric Functional Modeling is in essence a mathematical representation of the first-person observation characteristic of Eastern existential philosophies, where this representation facilitates convergence science through the equivalence of systems in this first person observation. One pathway described here towards this convergence is that functional states and the processes defining relationships between functional states are represented by geometries in functional state space. Geometric transforms (translation, reflection, etc.) of these geometries can enable truths from one region in conceptual space or in any other functional state spaces, to be used to discover truths in other regions. The relationship between this approach and the amazing proclivity of some Eastern mathematicians to discover new mathematical relationships without formal training, such as displayed by the mathematician Ramanujan [8], might be an interesting line of inquiry in terms of reviewing his work to detect any signature of this reasoning style. Srinivasa Ramanujan (22 December $1887-26$ April 1920)[8], as one example was a gifted layman who was able to derive a number of complex mathematical theorems largely without a formal education in mathematics. This paper proposes that Ramanujan and other such individuals might have developed a functional model of mathematics that enabled him to in essence develop an algorithm for creativity, that is, an algorithm for generalizing complex relationships and reusing them in other domains where they applied. What amounts to functional modeling has been proposed to be a fundamental part of the Vedic culture of which Ramanujan was a devoted follower. Functional modeling defines systems in terms of a set of discrete functions, and in terms of the states accessible through those functions (functional state space), so that the system can be describes as having dynamics governing its movement through that functional state space. By describing systems in terms of functions and the dynamics between those functions, complex systems can be understood even where the mechanisms by which those functions are implemented are unknown.

\section{Conclusions}

The assessment of the truth of a thing according to what that thing functions to achieve in this existence is part of Eastern existentialism, such as represented by the many-thousand year old Vedic tradition.

One core philosophy of such Eastern existential traditions is that the entire universe can be most reliably understood by looking inwards through first person observation and learning (developing the awareness) to observe the impact of the external world on our internal system. In the past science has largely ignored first person observations as the basis for research inquiry, though on closer inspection it usually plays a role [6]. But Human-Centric Functional Modeling facilitates a mathematical representation of first person observation in which truth is well-defined. By providing accurate first person observations, HCFM creates the potential for those first person observations to be used as a reliable scientific tool. For a great many problem domains, HCFM provides a clear way to validate or disprove theoretical models from first person observation of ones own awareness that can be performed independently of knowledge of any third party theories. HCFM is essentially the most generalizable possible approach to systems thinking. Where systems thinking approaches are often different and sometimes irreconcilable because they are based on the reasoning of one individual or another, HumanCentric Functional Modeling aims to become universal through looking inwards to the way all human beings perceive. Existential philosophical traditions such as yoga suggest that whether through our five senses, through our cognition, through our consciousness, or through other functional components of this human system, the external world functions to achieve some impact on this human organism, and that by sharpening our ability to observe this impact we can increase our capacity to perceive the truth of our own system and of the systems around us.

\section{References}

[1] Dodig Crnkovic, G. Dynamics of Information as Natural Computation. Information 2011, 2, 460477. https://doi.org/10.3390/info2030460 
[2] Williams, A. E. (n.d.). Human-Centric Functional Modeling and Evolutionary Biology. Retrieved from osf.io/preprints/africarxiv/sy4b6

[3] Williams, A. E. (n.d.). Functional State Spaces and their Formation in Systems from Biological Organisms to the Physical Universe. Retrieved from osf.io/preprints/africarxiv/cj463

[4] Williams, A. E. (2020, July 11). Human Intelligence and General Collective Intelligence as Phase Changes in Animal Intelligence. https://doi.org/10.31234/osf.io/dr8qn

[5] Williams, A.E. (2021c) Are wicked problems a lack of general collective intelligence? AI \& Soc (2021). https://doi.org/10.1007/s00146-021-01297-8

[6] Rigato, J., Rennie, S. M., and Mainen, Z. F. (2019). The overlooked ubiquity of first-person experience in the cognitive sciences. Synthese, 1-37

[7] Andy E. Williams, A Revolution in Systems Thinking?, Proceedings of the World Organization of Systems and Cybernetics (WOSC) 2021 Congress, Accepted (2021), link

[8] Kanigel, Robert (1991). The Man Who Knew Infinity: a Life of the Genius Ramanujan. New York: Charles Scribner's Sons. ISBN 978-0-684-19259-8.

[9] Gödel, K (1929). "Über die Vollständigkeit des Logikkalküls". Doctoral dissertation. University Of Vienna. The first proof of the completeness theorem.

[10] Williams, A. E. (2021, October 30). Comment in Reply to "On the complexity of extending the convergence region for Traub's method". https://doi.org/10.31730/osf.io/2zbyq 